\title{
Breath pentane as a marker for lipid peroxidation and adverse outcome in preterm infants
}

\author{
J A Nycyk, J A Drury, R W I Cooke
}

\begin{abstract}
Aim-To test the hypothesis that complications of neonatal intensive care are related to increased oxygen derived free radical activity, using breath pentane as a marker of lipid peroxidation.

Methods-Exhaled breath was collected daily from 57 ventilated preterm infants and pentane concentration measured by gas chromatography.

Results-High peak pentane exhalation was significantly associated with low gestational age, mortality, intraventricular haemorrhage and retinopathy of prematurity. Peak pentane was not significantly associated with the development of chronic lung disease.

Conclusions-The demonstration that pentane exhalation is related to the course of neonatal disease and its outcome is consistent with the hypothesis that lipid peroxidation is associated with these illnesses, and may contribute to their severity. If this is a causal relation, antioxidant treatments could prove useful in reducing their severity. Measurement of breath pentane might assist in the assessment of antioxidant strategies prior to more extensive clinical trials.

(Arch Dis Child Fetal Neonatal Ed 1998;79:F67-F69)
\end{abstract}

Keywords: oxygen derived free radicals; breath pentane; lipid peroxidation

Preterm infants receiving intensive care may sustain various complications such as intracranial haemorrhage and infarction (IVH), chronic lung disease (CLD), and retinopathy of prematurity (ROP). All of these have been attributed in part to tissue injury by oxygen derived free radicals.${ }^{1}$ This could either be due to excess generation of free radicals, or to immature antioxidant defence systems. Direct evidence for the generation of free radicals in biological systems is difficult to obtain, and measurement of products of oxidation are usually made to infer that excessive free radical activity is present. In preterm infants, raised concentrations of malondialdehyde, a product of lipid peroxidation, have been demonstrated

Department of Child Health,

Neonatal Unit, Liverpool Women's Hospital, Liverpool, L8 7SS

J A Nycyk

J A Drury

R W I Cooke

Correspondence to: Professor Cooke.

Accepted 14 January 1998 themselves contain dissolved pentane, and are peroxidised by the infant after administration, making interpretation of breath pentane levels difficult. ${ }^{45}$ These studies were also limited by small numbers, and the combination of death with other outcomes, such as CLD. ${ }^{6}$

We used a new method ${ }^{7}$ for the measurement of pentane in infant breath to test the hypothesis that complications of neonatal intensive care are related to increased lipid peroxidation.

\section{Methods}

All preterm infants of less than 33 weeks of gestation admitted to the neonatal intensive care unit at Liverpool Women's Hospital between August 1995 and August 1996 were included in the study provided that they were mechanically ventilated on day one, and their parents allowed their infant to be included. The project had the approval of the local paediatric research ethics committee. Samples of exhaled breath were collected from infants on each of the first five days while the infant remained intubated. All infants received artificial pulmonary surfactant (ALEC, Britannia Pharmaceuticals, UK).

Data on clinical and demographic variables were collected prospectively. Cranial ultrasound scans were routinely made in the first 24 hours after birth, and at regular intervals until discharge. Scans were graded according to the system described by Papille. ${ }^{8}$ Regular screening for retinopathy was undertaken by one paediatric ophthalmologist, and disease staged according to the International Classification of Diseases. ${ }^{9}$ Chronic lung disease was defined as oxygen dependency at 36 weeks of postmenstrual age (CLD36). Survivors were those infants alive at this time.

Breath samples were collected from infants after a 5 minute washout period, breathing hydrocarbon free air/oxygen mixture (AS80 air purifier, Signal Instruments). Infants were

Table 1 Clinical and outcome variables for all subjects

\begin{tabular}{lc}
\hline$N=57$ & $\begin{array}{c}\text { Median (range) or } \\
\text { number (\%) }\end{array}$ \\
\hline Gestation (weeks) & $27(22-32)$ \\
Birthweight (g) & $892(520-1870)$ \\
Sex (M/F) & $28: 29$ \\
Mode of delivery (vaginal:C-section) & $28: 29$ \\
Survivors & $44(77 \%)$ \\
Chronic lung disease & $30(53 \%)$ \\
Intraventricular haemorrhage & $9(16 \%)$ \\
Grade 1 & $8(14 \%)$ \\
Grade 2 & $6(11 \%)$ \\
Grade 3 & $9(16 \%)$ \\
Grade 4 & $32(56 \%)$ \\
All grades & \\
Retinopathy of prematurity & $11(19 \%)$ \\
Stage 1 & $11(19 \%)$ \\
Stage 2 & $2(4 \%)$ \\
Stage 3 & $24(42 \%)$ \\
All stages &
\end{tabular}
in the urine and plasma of sick infants, using the thiobarbituric acid test, although the test is not very specific. ${ }^{23}$ Peroxidation of cell membrane lipids gives rise to ethane and pentane by a process of $\beta$ scission, and measurement of these alkane gases in the exhaled breath has been advocated as a more specific indicator of lipid peroxidation by freed radicals in vivo. Previous work using this technique in preterm infants has been complicated by the use of intravenous lipid emulsion infusions, which 


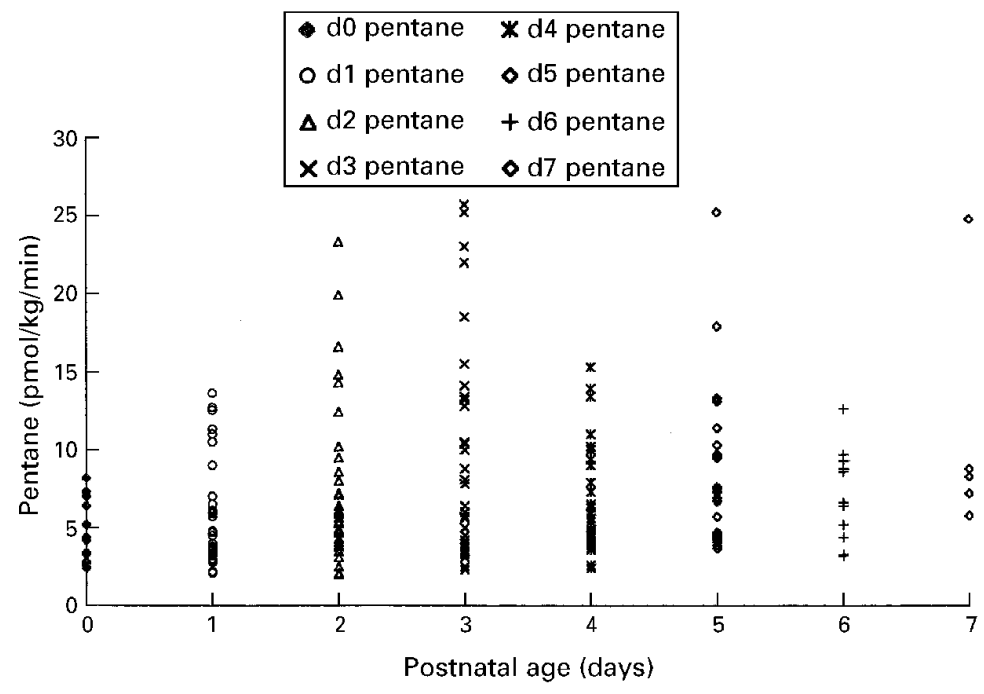

Figure 1 Pentane concentrations for all subjects during the first eight days after birth.

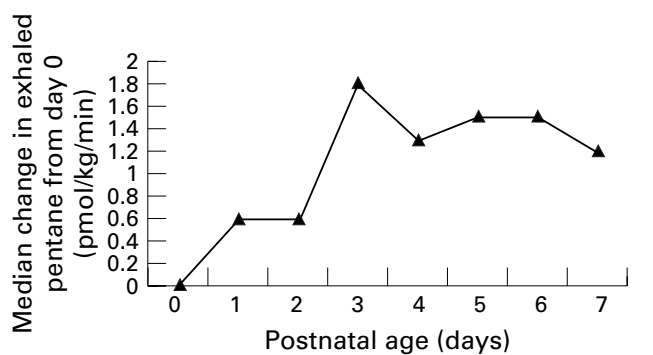

Figure 2 Median difference between pentane concentrations exhaled on consecutive days.

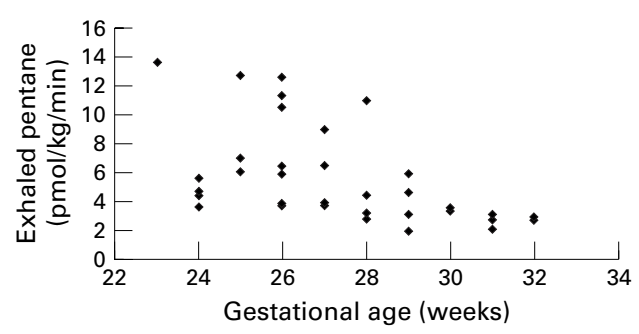

Figure 3 Day 1 pentane concentrations plotted against gestational age $(n=34)$.

hand ventilated using an anaesthetic circuit with a non-rebreathing valve, and using rates, pressures and oxygen concentrations identical with those being used before the collection period. A one minute sample was collected in a 1 litre Tedlar bag, and a measured amount of cis-2-pentene added as an internal standard. A sample of the total expired gas collection was adsorbed on to Carbotrap in a sample tube, before thermal desorption, cryofocusing, and analysis on a gas chromatograph (Chrompack $9001 \mathrm{GC})$ with a capillary column $\left(\mathrm{Al}_{2} \mathrm{O}_{3} / \mathrm{KCl}\right.$ PLOT). All measurements were made in duplicate, and the mean value used. Pentane measurements were expressed as $\mathrm{pmol} / \mathrm{kg} / \mathrm{min}$. The interassay coefficient of variance was $3.6 \%$ and the intra-assay $1.7 \%$. The method has been described in detail elsewhere. ${ }^{7}$

Spearman's correlation coefficient and the Mann-Whitney U test were used for the analysis of the results.
Table 2 Median peak pentane measured according to clinical and outcome variables

\begin{tabular}{lrl}
\hline & $\begin{array}{l}\text { Median maximum pentane } \\
\text { [pmol/kg/min] (IQ range) }\end{array}$ & p Value \\
\hline Male & $7.9(4.7-12.0)$ & \\
Female & $10.0(4.7-13.4)$ & 0.72 \\
Vaginal delivery & $12.5(6.4-15.5)$ & 0.02 \\
Caesarean section & $5.9(4.7-10.1)$ & \\
Singleton & $9.5(4.7-14.1)$ & 0.71 \\
Multiple & $7.8(4.7-12.5)$ & \\
Antenatal steroids & $9.7(4.7-13.2)$ & 0.69 \\
No steroids & $7.1(4.6-10.9)$ & 0.004 \\
Died & $11.0(10.1-15.0)$ & 0.015 \\
Survived & $5.8(4.4-9.9)$ & \\
IVH & $10.1(4.9-14.3)$ & 0.53 \\
No IVH & $6.4(4.1-9.2)$ & 0.034 \\
CLD36 & $6.1(4.8-10.1)$ & \\
No CLD & $5.8(3.7-10.7)$ & \\
ROP & $6.8(4.7-13.2)$ & \\
No ROP & $5.3(3.5-7.8)$ & \\
\hline Table 3 Median pentane measured on day 1 according to \\
outcome variables & & \\
\hline
\end{tabular}

\begin{tabular}{lll}
\hline & $\begin{array}{l}\text { Median pentane level, day } 1 \\
(\text { pmol/kg/min) }(\text { IQ range) }\end{array}$ & p Value \\
\hline Died & $5.3(3.8-11.1)$ & \\
Survived & $4.3(3.2-6.9)$ & 0.19 \\
CLD36 & $6.1(3.7-9.8)$ & \\
No CLD & $3.2(2.8-4.2)$ & 0.01 \\
ROP & $7.3(3.8-10.6)$ & 0.03 \\
No ROP & $3.5(2.9-5.6)$ & \\
IVH & $5.8(3.8-10.7)$ & 0.01 \\
No IVH & $3.7(2.9-5.8)$ & \\
\hline
\end{tabular}

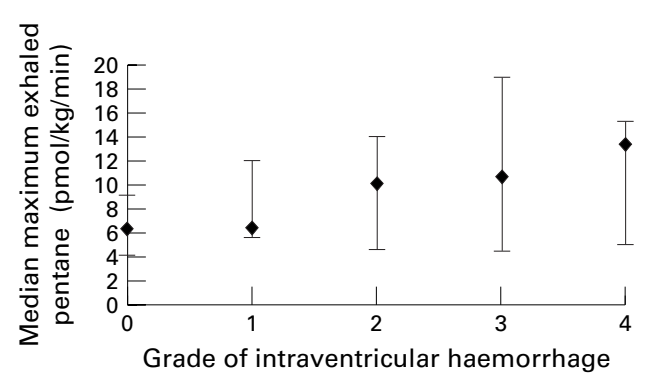

Figure 4 Peak pentane concentration by grade of intraventricular haemorrhage; median and interquartile range

\section{Results}

Fifty seven infants were included in the study. Demographic, clinical, and outcome data are presented in table 1 . One hundred and fifty two estimations of breath pentane were made in these infants over 5 successive days after birth. Incomplete data in some infants related to early death, extubation, or to technical problems. Pentane concentrations rose significantly from a median value of $3.35 \mathrm{pmol} / \mathrm{kg} / \mathrm{min}$ on day 0 , to a median peak of $7.80 \mathrm{pmol} / \mathrm{kg} / \mathrm{min}$ on day 3 $(p=0.001)$ (fig 1). Because pentane production is affected by within subject factors and between subject factors, and the absence of some values may have been related to pentane concentrations in babies who died, we also included data showing the median change between days (calculated from day 0) (fig 2). There was a significant inverse correlation between increasing gestational age and pentane exhalation on day 1 $(\mathrm{r}=-0.67, \mathrm{p}<0.0001 ; \mathrm{n}=34$ ) (fig 3).

Peak pentane exhalation did not differ significantly with sex, use of antenatal steroids or if the infant was a singleton or twin. Infants born by the vaginal route had significantly higher values than those delivered by caesarean 
section $(\mathrm{p}=0.02)$ (table 2). A high peak pentane exhalation was also significantly associated with mortality $(p=0.004)$, intraventricular haemorrhage $(p=0.015)$, and ROP $(p=0.034)$. Pentane concentrations were highest in those infants with the largest grade of cerebral haemorrhage/ infarction (fig 4). There was no significant association between peak pentane exhalation and the development of chronic lung disease $(p=0.53)$ (table 2). Significant associations were, however, seen between intraventricular haemorrhage, ROP, and CLD and pentane concentrations on day 1 (table 3 ).

\section{Discussion}

This study shows that pentane exhalation in sick preterm infants is significantly related to the course of neonatal disease and, to some extent, its outcome. This is certainly consistent with the hypothesis that lipid peroxidation is associated with these illnesses, and may even contribute to their severity. Respiratory distress syndrome is known to worsen over the first 72 hours after birth. This corresponds temporally with an increase in the numbers of macrophages and neutrophils entering the lung, and to the release of cytokines and proteases, at least in part due to the generation of superoxide by the cells' respiratory burst. ${ }^{10}$

It also corresponds to the time of exposure of the lung to maximum inspired oxygen concentrations, although we have not been able to demonstrate a significant correlation between breath pentane and inspired oxygen concentration. $^{7}$ The finding of a peak in pentane production corresponding to a peak in cellular activity in the lung suggests that the latter causes a degree of peroxidation. Previous studies have shown that peak pentane production in sick preterm infants is associated with poor outcome, a measure combining CLD (at 28 days) with death in a single variable. ${ }^{4}$ In this study, when these variables were considered separately, peak pentane was not significantly related to CLD, although it was with mortality.

Several factors may have contributed to this finding. The definition used for CLD was more severe than that used before, as only 13 infants were not oxygen dependent at 28 days due to their immaturity and severity of respiratory disease. If this small group of infants was examined, there remained no significant difference in exhaled pentane between these babies and those who were oxygen dependent at 28 days. The incidence of intracranial haemorrhage and infarction was also high, and haemorrhage was strongly associated with peak pentane concentrations, which may have obscured any pulmonary pentane production. The fact that CLD was related to pentane values on day 1 , before most cerebral haemorrhage, is consistent with this hypothesis. The significant association of all grades of retinopathy with pentane production may simply reflect an association with disease severity in general, or reflect a state of increased free radical generation in the infant in general during the first few days after birth. Pentane production was certainly highest in infants with the lowest gestational age, possibly because of poorly developed antioxidant defences.
The method used to measure expired pentane in this study permitted frequent and relatively simple trapping and analysis of the gas without the need to remove carbon dioxide and water first. The use of an internal standard avoided errors during transfer of the specimens to the gas chromatograph.

The origin of the pentane in exhaled breath is not entirely clear. Pentane is generated from linoleic acid by peroxidation, but it may also be generated by bacterial action in the gut. It has been assumed that a considerable proportion of the pentane found in breath is from peroxidation of cell membranes in the lung, although it is not possible to separate this from pentane generated elsewhere. Infusions of lipids as part of total parenteral nutrition are also sources of breath pentane, both because they contain dissolved pentane from spontaneous peroxidation during storage, and they generate further pentane due to their peroxidation during metabolism. This study was confined to the first week after birth when no parenteral lipid infusions were being given, to avoid the contribution of lipid to breath pentane.

If the lipid peroxidation observed in sick preterm infants is causally related to the complications of intensive care that they experience, antioxidant treatment could prove useful in reducing their severity. Vitamin $\mathrm{E}$ has been used to prevent cerebral haemorrhage, and vitamin $\mathrm{A}$ and superoxide dismutase to prevent chronic lung disease with varying degrees of success. ${ }^{11-13}$ In practice a combination of agents, as occurs in vivo, may provide a better solution than monotherapies. Measurement of breath pentane might assist in the assessment of suitable antioxidant strategies prior to more extensive clinical trials.

1 Saugstadt OD. Hypoxanthine as an indicator of hypoxia: its role in health and disease through free-radical production. Pediatr Res 1988;23:143-50.

2 Inder TE, Graham P, Sanderson K, Taylor BJ. Lipid peroxidation as a measure of oxygen free radical damage in the dation as a measure of oxygen free radical damage in the very

3 Schlenzig JS, Bervoets K, von Loewenich V, Bohles H. Urinary malondialdehyde in preterm neonates; is there a relationship to disease entities of neonatal intensive care? Acta Paediatr 1993;82:202-5

4 Pitkanen OM, Hallman M, Andersson SM. Correlation of free oxygen radical-induced lipid peroxidation with outcome in very low birthweight infants. 7 Pediatr 1990;116:760-4.

5 Pitkanen OM, Hallman M, Andersson SM. Generation of free radicals in lipid emulsion used in parenteral nutrition. Pediatr Res 1991;29:56-9.

6 Varsila E, Pitkanen O, Hallman M, Andersson S. Immaturity-dependent free radical activity in premature infants. Pediatr Res 1994;36:55-9.

7 Drury JA, Nycyk JA, Cooke RWI. Pentane measurement in ventilated infants using a commercially available system.

Free Radical Biol Med 1997;22:895-900.
8 Papille LA, Birskin J, Birskin R, Koffler H. Incidence and evolution of subependymal and intraventricular haemorrhage: a study of infants with birthweights less than 1500 gm. F Pediatr 1978;92:529-34

9 Committee fo the classification of retinopathy of prematurity. International classification of ROP. Pediatrics 1984;74:127-33.

10 Arnon S, Grigg J, Silverman M. Pulmonary inflammatory cells in ventilated preterm infants: effect of surfactant treatment. Arch Dis Child 1993;69:44-8.

11 Chiswick ML, Johnson M, Woodhall C. Protective effect of vitamin $\mathrm{E}$ (dl-alpha-tocopherol) against intraventricular haemorrhage in premature babies. BMF 1983;287:81-4.

12 Shenai JP, Kennedy KA, Chytil F, Stahlman MT. Clinical trial of vitamin A supplementation in infants susceptible to
bronchopulmonary dysplasia. F Pediatr 1987;111:269-77.

13 Rosenfeld W, Evans H, Concepcion L, Jhaveri R, Schaeffer H, Friedman A. Prevention of bronchopulmonary dyspla$\mathrm{H}$, Fria by administration of bovine superoxide dismutase in preterm infants with respiratory distress syndrome. F Pedipreterm infants with 